\title{
Analytical expression and experimental validation of the Brillouin gain spectral broadening at any sensing spatial resolution
}

\author{
Mehdi Alemª, Marcelo A. Soto ${ }^{\mathrm{a}}$, Moshe Tur ${ }^{\mathrm{a}, \mathrm{b}}$, and Luc Thévenaz ${ }^{\mathrm{a}}$ \\ ${ }^{a}$ EPFL Swiss Federal Institute of Technology, Institute of Electrical Engineering \\ SCI STI LT, Station 11, CH-1015 Lausanne, Switzerland; \\ ${ }^{\mathrm{b} S}$ School of Electrical Engineering, Tel Aviv University, Ramat Aviv 69978, Israel; \\ *E-mail: Luc.Thevenaz@EPFL.CH
}

\begin{abstract}
A novel and simple analytical expression to describe the Brillouin gain spectral broadening as a function of the spatial resolution in time-domain Brillouin distributed fiber sensors is deduced. The proposed model is experimentally validated using a pump-probe Brillouin sensing setup and also compared with numerical and approximate results. In addition, a compact mathematical form is presented for the peak gain reduction resulting from incomplete acoustic-wave activation in Brillouin sensors with short spatial resolution. Both mathematical expressions can be used together to quantitatively predict the impact of the spatial resolution on the signal-to-noise ratio and frequency uncertainty of the sensor.
\end{abstract}

Keywords: Distributed fiber sensor, stimulated Brillouin scattering, Brillouin gain spectrum, spectral broadening.

\section{INTRODUCTION}

Brillouin optical time-domain analysis (BOTDA) fiber sensors are extensively employed for distributed measurements of temperature and strain due to their unique advantages such as long sensing range and relatively short spatial resolution. BOTDA sensors rely on the stimulated Brillouin scattering (SBS) interaction of two counter-propagating light signals, a continuous-wave $(\mathrm{CW})$ probe and a pulsed-light pump with a frequency offset being scanned around the Brillouin frequency shift (BFS) of the fiber, i.e. $\sim 11 \mathrm{GHz}$. On the one hand, the performance of Brillouin distributed sensors is strongly dependent on the spectral features of the Brillouin gain spectrum (BGS) ${ }^{1}$, such as width and the maximum gain value, which scales the signal-to-noise ratio (SNR) of the system ${ }^{1}$. Hence, it is essential for system performance prediction and optimization to provide simple but sufficiently accurate mathematical expressions for the Brillouin gain reduction and spectral broadening. On the other hand, the Brillouin gain spectrum of a sensing system is determined by the pump light characteristics, such as pulse shape and duration ${ }^{2}$. In almost all time-domain Brillouin fiber sensors, a rectangular pulse with very sharp rise and fall times is used to modulate the pump laser light in order to avoid the detrimental spectral broadening of the pump light resulting from self-phase modulation (SPM), and thus avoiding the undesired reduction in the Brillouin gain ${ }^{3}$.

For rather low pump powers when the small-signal gain holds ${ }^{1}$ and the pump depletion due to modulation instability (MI) does not impact ${ }^{4}$, the measured gain due to the Brillouin interaction can be expressed by $g_{B} I_{P} \Delta L \exp (-\alpha L)$, where $g_{B}$ is the Brillouin gain, $I_{P}$ is the pump intensity, $\Delta L$ is the pump pulse length, $\alpha$ is the fiber attenuation and $L$ is the fiber length. The Brillouin gain $g_{B}$ is frequency dependent and modified by the pump pulse shape. When a CW light is used as pump, the Brillouin gain shows a Lorentzian shape as follows ${ }^{5}$ :

$$
g_{\mathrm{B}}(\Omega)=g_{0} \frac{\Gamma^{2}}{\Gamma^{2}+\Omega^{2}},
$$

where $\Omega=2 \pi f$ is the frequency detuning around the Brillouin frequency shift $\Omega_{\mathrm{B}}, g_{0} \sim 3 \times 10^{-11} \mathrm{~m} / \mathrm{W}$ is the maximum Brillouin gain, and $\Gamma=\Gamma_{\mathrm{B}} / 2$ is the half-width at half maximum (HWHM) of the Brillouin gain spectrum in which $\Gamma_{\mathrm{B}} \sim 2 \pi \times 30 \mathrm{Mrad} / \mathrm{s}$ is its full-width at half maximum (FWHM) in the $1550 \mathrm{~nm}$ transmission window. In this paper the amplitude reduction and spectral broadening of the Brillouin gain spectrum are thoroughly investigated and modelled as a function of the spatial resolution of the sensor. The proposed mathematical expressions, combined with the model presented in Ref. 1, can be reliably used to evaluate the impact that the spatial resolution has on the SNR and ultimately fully predict the performance of the sensor, e.g. in terms of distance range and measurand accuracy. 


\section{GAIN REDUCTION}

When the pump light is shaped as a pulse, the Brillouin gain spectrum is obtained from the convolution of the Lorentzian-shaped natural Brillouin gain spectrum given by Eq. (1) and the normalized power spectral density (PSD) of the pump pulse ${ }^{6}$. In the case of using a rectangular pulse (which is the optimal shape to avoid the SPM-induced spectral broadening and gain reduction), the resulting BGS shape can be calculated analytically and represented in a closed-form expression ${ }^{7}$. The normalized PSD of a rectangular pulse of duration $T$ is $S_{p}(f)=T \operatorname{sinc}^{2}(T f)$ where $\int_{-\infty}^{+\infty} S_{p}(f) d f=1$. Therefore, the Brillouin gain spectrum $g_{T}(\Omega)$ generated by a rectangular-shaped pump pulse is derived from the convolution between $g_{\mathrm{B}}$ and $S_{p}$ as follows:

$$
g_{T}(\Omega)=g_{\mathrm{B}}(\Omega)\left(1-\frac{\left(\Gamma^{2}-\Omega^{2}\right)\left(1-e^{-\Gamma T} \cos (T \Omega)\right)+2 \Gamma \Omega e^{-\Gamma T} \sin (T \Omega)}{\Gamma T\left(\Gamma^{2}+\Omega^{2}\right)}\right) .
$$

Although a similar expression was proposed by A.Fellay et al. ${ }^{7}$ (inexact, but later corrected in his PhD dissertation doi:10.5075/epfl-thesis-2728) and F. Ravet et al. ${ }^{8}$, here we present the gain spectrum in a more tangible and compact form to highlight its reduction due to pump modulation. A simple analytical expression is derived for the peak Brillouin gain (i.e. for $\Omega=0$ ), reducing from $g_{0}$ for $\mathrm{CW}$ pump to the following value for a rectangular pump pulse of duration $T$ :

$$
g_{\max }=g_{0}\left(1-\frac{1-e^{-\Gamma T}}{\Gamma T}\right) .
$$

Based on Eq. (3), the reduction in the maximum Brillouin gain can be expressed in terms of the spatial resolution $\Delta L=$ $T v_{g} / 2$, where $v_{g}$ is the group velocity of the pump pulse. This reduction factor is essential to estimate precisely the SNR of the sensing system ${ }^{1}$. Fig. 1(a) shows the Brillouin gain spectrum given in Eq. (2) for different values of pump pulsewidth (proportional to the spatial resolution). As an example, improving the spatial resolution from $2 \mathrm{~m}(T=20 \mathrm{~ns})$ down to $1 \mathrm{~m}(T=10 \mathrm{~ns})$ reduces the measurement SNR by $\sim 5 \mathrm{~dB} ; 3 \mathrm{~dB}$ are due to the pulse-width reduction, and $\sim 2 \mathrm{~dB}$ additional penalty in the SNR are due to the Brillouin gain reduction caused by the weaker activation of the acoustic wave. Fig. 1(b) depicts the gain factor $g_{\max } / g_{0}$ given by Eq. (3), indicating that the Brillouin gain reduces drastically for pulses shorter than the acoustic wave response time $(\sim 11 \mathrm{~ns})$, corresponding to spatial resolutions shorter than $1 \mathrm{~m}$.
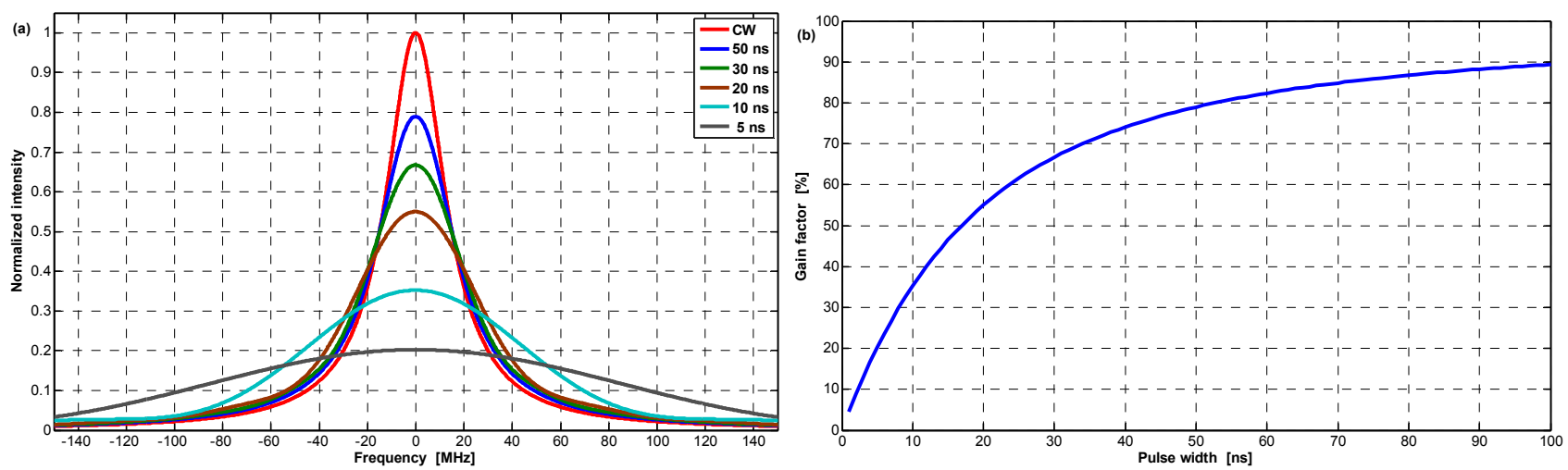

Figure 1. (a) Brillouin gain spectrum for different values of spatial resolution or pump pulse duration normalized to the CW maximum Brillouin gain $g_{0}$. (b) Brillouin gain reduction factor versus the pump pulse width with respect to the $\mathrm{CW}$ maximum Brillouin gain $g_{0}$. The FWHM of the Lorentzian gain spectrum is assumed to be $30 \mathrm{MHz}$.

\section{SPECTRAL BROADENING}

Note that not only the gain reduction due to a spatial resolution improvement affects the system performance but also the broadening of the gain spectrum increases the frequency uncertainty of the sensor ${ }^{1}$. In order to quantify the effect of spectral broadening on the system performance it is compulsory to find the FWHM of the BGS broadened by the pump pulse spectrum. This can be performed by solving the FWHM equation $g_{T}(\Omega)=g_{\max } / 2$ for $\Omega$, where $g_{T}$ is the widened spectrum given in Eq. (2). After a lengthy derivation it results in a quasi-polynomial equation for $\Omega$ as follows:

$$
\begin{aligned}
\Gamma^{4}\left(1-\Gamma T+e^{-\Gamma T}\right)-2 \Gamma^{2}\left(2-e^{-\Gamma T}\right) \Omega^{2}+\left(\Gamma T-1+e^{-\Gamma T}\right) \Omega^{4} \\
=2 e^{-\Gamma T} \Gamma^{2}\left(\Gamma^{2}+\Omega^{2}\right) \cos \left(T \Omega+\tan ^{-1}\left(\frac{2 \Gamma \Omega}{\Gamma^{2}-\Omega^{2}}\right)\right) .
\end{aligned}
$$


Eq. (4) gives no closed-form solution for $\Omega$; however, it is possible to provide an approximate but still analytical solution for the equation by substituting the cosine function by its either maximum or minimum. The only real and positive solution is obtained when the cosine function is substituted by its maximum and thus, the equation reduces to:

$$
\Gamma^{4}\left(1-\Gamma T-e^{-\Gamma T}\right)-4 \Gamma^{2} \Omega^{2}+\left(\Gamma T-1+e^{-\Gamma T}\right) \Omega^{4}=0 .
$$

This equation has one real and positive solution which gives the half-width at half-maximum of the broadened Brillouin gain spectrum. This way, the FWHM of the Brillouin gain spectrum of a pulsed pump is obtained from the CW Brillouin width using a broadening factor $B F$ such that $\mathrm{FWHM}_{\mathrm{Pulse}}=B F \times \mathrm{FWHM}_{\mathrm{CW}}$ and given by the following expression:

$$
B F=\sqrt{\frac{2+\sqrt{4+\psi^{2}}}{\psi}},
$$

in which $\psi=\Gamma T-1+e^{-\Gamma T}$. This novel expression for the BGS spectral broadening is useful in any optical fiber system utilizing Brillouin scattering generated by a rectangular pump pulse. For instance, when the spatial resolution is $2 \mathrm{~m}$ or equivalently the pulse width is $T=20 \mathrm{~ns}$, according to Eq. (6) the broadening factor is calculated to be $\sim 2$, i.e. the FWHM of the Brillouin spectrum for a BOTDA with $2 \mathrm{~m}$ spatial resolution turns out to be $\sim 60 \mathrm{MHz}$, which is in good agreement with the experimental observations reported in the literature ${ }^{7}$ and with the experimental results presented in the next section. This analytical formula for the Brillouin bandwidth given by Eq. (6) can also be used as a tool to improve the figure of merit (FoM) of Brillouin distributed sensors and compare different configurations of sensors ${ }^{1}$. It is also worth evaluating the inverse proportionality of the spectral width with respect to the pulse duration. Considering the fact that time and frequency are inversely proportional, there is a tendency to obtain the spectral width of the Brillouin gain simply as a factor of $T^{-1}$. It can be shown that such an approximation is valid for short pulses where $T \ll \Gamma^{-1}$ or equivalently, for sharp spatial resolution Brillouin sensors. When $\Gamma T \ll 1$, the exponential function $e^{-\Gamma T}$ can be approximated by the first three terms of its Taylor series so that $\psi \approx \Gamma^{2} T^{2} / 2$. Substituting $\psi$ in Eq. (6), the FWHM of the Brillouin gain spectrum expressed in $\mathrm{Hz}$ is obtained as follows:

$$
\text { FWHM }_{\text {Pulse }}=\frac{2 \sqrt{2}}{\pi T} \approx \frac{0.9}{T}
$$

As we will see in the next section, the simple expression in Eq. (7) is only valid for sharp spatial resolution Brillouin distributed sensors, in which the pulse duration $T$ is shorter than the acoustic wave time constant $\Gamma^{-1} \approx 11 \mathrm{~ns}$. Hence, for standard BOTDA sensors with meter-range spatial resolutions, Eq. (7) results in an unreliable value for the Brillouin spectral width and thus, it must be calculated from the proposed expression in Eq. (6) to secure a good accuracy.

\section{EXPERIMENTAL VALIDATION}

The experimental setup for validating the proposed mathematical model is simply a standard BOTDA system that utilizes a pump-probe approach for measuring the Brillouin interaction. On the pump side, the CW light generated by a DFB laser is modulated using an electro-optic modulator to generate rectangular pulses with sharp rising and falling times (limited in this case by the pulse generator to $300 \mathrm{ps}$ ). On the probe side, the $\mathrm{CW}$ light of the laser is modulated by an RF tone, generating a two-sideband probe that is used to scan the Brillouin gain spectrum covering a spectral range of $300 \mathrm{MHz}$, which is 10 times more than the FWHM of the natural Lorentzian-shaped Brillouin spectrum.
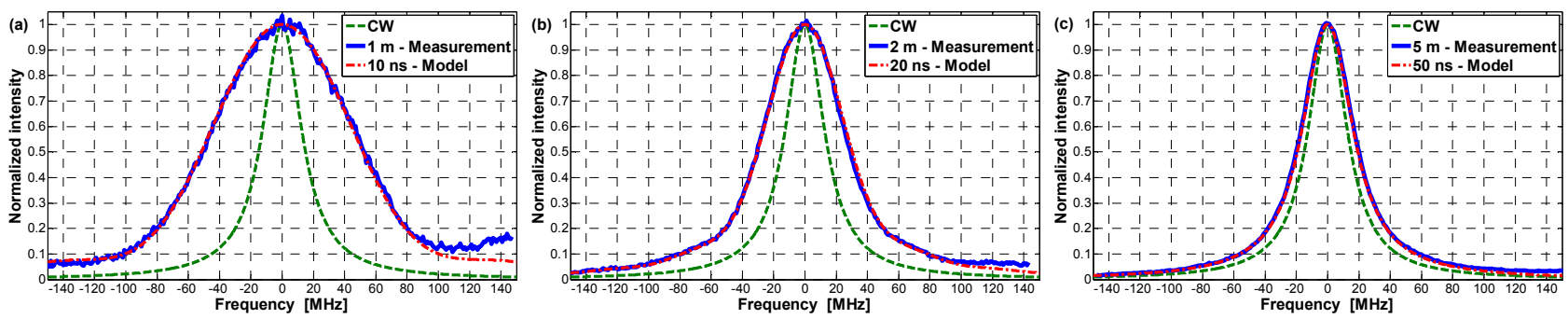

Figure 2. Normalized Brillouin gain spectrum for different values of spatial resolution (a) $1 \mathrm{~m} \mathrm{(10} \mathrm{ns} \mathrm{pulse),} \mathrm{(b)} 2 \mathrm{~m}$ (20 ns pulse), and (c) $5 \mathrm{~m} \mathrm{(50} \mathrm{ns} \mathrm{pulse).} \mathrm{Measurements} \mathrm{(blue} \mathrm{lines)} \mathrm{are} \mathrm{compared} \mathrm{with} \mathrm{the} \mathrm{analytical} \mathrm{model} \mathrm{in} \mathrm{Eq.} \mathrm{(2)} \mathrm{(red} \mathrm{lines),}$ and, as a reference, with the natural Lorentzian-shaped Brillouin spectrum of Eq. (1) (green lines). The FWHM of the Lorentzian gain spectrum of the fiber under test is set in this case to $30 \mathrm{MHz}$. 
Fig. 2 compares the measured Brillouin gain spectrum with the analytical model presented in Eq. (2), demonstrating an excellent agreement between the experiment and theory. For a better visibility of the spectral broadening, the Lorentzian spectrum corresponding to a $\mathrm{CW}$ pump is depicted in each plot (green dashed line). As it is clear from Fig. 2(a) and Fig. 2(b), a substantial increase in the Brillouin spectral width (in this case from $60 \mathrm{MHz}$ up to $105 \mathrm{MHz}$ ) occurs when the pulse duration approaches the acoustic-wave response time; while for longer pump pulses, the difference between the resulting gain spectrum and the natural Lorentzian one turns moderate, as depicted in Fig. 2(c).

In Fig. 3, the FWHM of the Brillouin gain spectrum is depicted as a function of the pump pulse width. The experimental measurements of the FWHM are shown by red squares, while the analytical model given in Eq. (6) is plotted by a red continuous line. The figure demonstrates that experimental results match very well the description provided by the proposed model. In addition, the figure also shows (dashed blue line) the FWHM obtained by solving Eq. (4) numerically. It can be observed that the approximate analytical solution of Eq. (4) given in Eq. (6) is indeed a perfect approximation for the FWHM of the BGS. In contrast, the figure also verifies that the approximate FWHM (green dashed line) being inversely proportional to the pulse duration and represented by Eq. (7) is not accurate enough to describe the FWHM of the BGS measured in standard meter-scale BOTDA sensors.

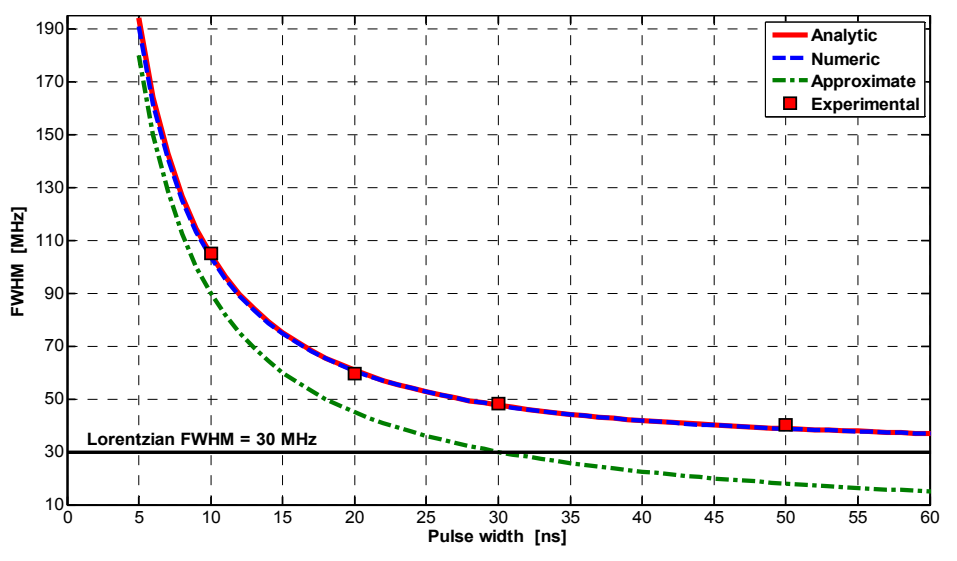

Figure 3. FWHM of the Brillouin gain spectrum versus pump pulse duration; comparison of the measurement, numerical exact value in Eq. (4), analytical model in Eq. (6), and approximate value in Eq. (7).

In conclusion, a mathematical model for the Brillouin gain spectrum resulting from a BOTDA sensor has been presented, along with analytical close-form expressions describing the spectral broadening and gain reduction resulting from the shorter acoustic-wave activation when aiming at spatial resolution of a few meters. The model has been validated by numerical solutions and experimental results obtained from a standard BOTDA system. The spectral broadening and gain amplitude factors introduced in this work constitute a unique and simple tool for a full prediction of the impact of spatial resolution on the performance and figure-of-merit of Brillouin distributed optical fiber sensors.

The authors acknowledge the support from the Swiss Commission for Technology and Innovation (Project 18337.2 PFNMNM). The work of M. Tur was supported in part by the Israel Science Foundation (grant No. 1380/12).

\section{REFERENCES}

[1] Soto, M. A., and Thévenaz, L., "Modeling and evaluating the performance of Brillouin distributed optical fiber sensors," Opt. Express 21(25), 31347-31366 (2013).

[2] Smith, J., Brown A., DeMerchant M., and Bao, X., "Pulse width dependance of the Brillouin loss spectrum," Optics Communications 168(5), 393-398 (1999).

[3] Foaleng, S. M., Rodríguez-Barrios, F., Martin-Lopez, S., González-Herráez, M., and Thévenaz, L., "Detrimental effect of self-phase modulation on the performance of Brillouin distributed fiber sensors," Opt. Lett. 36(2), 97-99 (2011).

[4] Alem, M., Soto, M. A., and Thévenaz, L., "Analytical model and experimental verification of the critical power for modulation instability in optical fibers," Opt. Express 23(23), 29514-29532 (2015).

[5] Niklès, M., Thévenaz, L., and Robert, P. A., "Brillouin gain spectrum characterization in single-mode optical fibers," Journal of Lightwave Technology 15(10), 1842-1851 (1997).

[6] Lichtman, E., Waarts, R. G., and Friesem, A. A., "Stimulated Brillouin scattering excited by a modulated pump wave in single-mode fibers," Journal of Lightwave Technology 7(1), 171-174 (1989).

[7] Fellay, A., Thévenaz, L., Facchini, M., Niklès, M., and Robert, P. A., "Distributed sensing using stimulated Brillouin scattering: towards ultimate resolution," in Optical Fiber Sensors (OSA Digest), paper OWD3 (1997).

[8] Ravet, F., Bao, X., Zou, L., Yu, Q., Li, Y., Kalosha, V., and Chen, L., "Accurate strain detection and localisation with the distributed Brillouin sensor based on phenomenological signal processing approach," Proc. SPIE 6176, 61761C (2006). 